\title{
Une représentation 3D de la Transformée de Fourier Discrète
}

\author{
Jean-Claude Pissondes
}

\author{
Département Réseaux et Télécoms, IUT de Vélizy, \\ 10-12 Avenue de l'Europe 78140 Vélizy \\ jcpissondes@aim.com
}

Article soumis le 19-04-2016 et accepté le 22-09-2016

\begin{abstract}
Résumé-Dans cet article, on présente la forme augmentée d'un projet tuteuré proposé à des étudiants en DUT Réseaux et Télécoms (R\&T) sur la Transformée de Fourier Discrète (TFD). Dans une première partie, on étudie le principal acteur de la TFD, l'exponentielle complexe $W_{N}^{m n}=\exp (-2 i \pi m n / N)$ : on introduit une « représentation de Fresnel 3D » des coefficients $W_{N}^{m n}$ qui conduit à des figures dans l'espace en forme d'" escalier en colimaçon ». Ces figures 3D exhibent clairement les symétries et propriétés des $W_{N}^{m n}$ conduisant aux propriétés de la TFD. Dans un second temps, on effectue le même travail sur les produits $s(n) W_{N}^{m n}$ pour un signal $s(n)$ donné, dont la somme sur $n$ conduit aux différents éléments $X(m)$ de la TFD. D'une part, on observe sur les « escaliers » les propriétés usuelles et moins courantes de la TFD puis, comment la TFD « sélectionne " les fréquences caractéristiques du signal. D'autre part, on verra comment lire amplitude et phase pour une fréquence donnée et un signal donné, en particulier en présence d'un « retard ». Enfin, on indiquera les difficultés rencontrées par les étudiants et on fera un bref bilan des quelques projets déjà réalisés.
\end{abstract}

Mots clés-Transformée de Fourier discrète, Transformée de Fourier Rapide, TFD, FFT, Traitement du signal

\section{INTRODUCTION}

\section{A. Importance de la TFD}

La Transformée de Fourier Discrète (TFD) est omniprésente dans un cursus en Réseaux et Télécommunications, en DUT et ultérieurement : dès qu'une «machine »- un oscilloscope numérique en mode Maths ou un calculateur (un « ordinateur ») - exécute et affiche le spectre d'un signal, elle réalise une TFD. Il est donc important, pour un étudiant en Télécoms, de connaître ses principales propriétés. Parmi celles-ci, certaines d'entre elles permettent la mise en œuvre, par la machine, d'algorithmes de calcul rapide qui conduisent à la notion de Transformée de Fourier Rapide : la FFT, pour Fast Fourier Transform, qu'il convient de ne pas assimiler d'emblée à la TFD. De plus, la TFD présente, par construction, des différences notables avec la Transformée de Fourier étudiée en cours de Mathématiques, différences qu'un bon technicieningénieur en Télécoms doit aussi connaître. Le but de cette étude, proposée comme projet tuteuré depuis 2013, est donc de bien comprendre les spécificités de la TFD en utilisant des moyens aussi simples que possible et visuellement explicites. Notre étude repose principalement sur une utilisation judicieuse de la formule d'Euler $\exp (i x)=\cos x+i \sin x$ et sur la périodicité de $\exp (i x)$.

\section{B. Quelques rappels et originalité du projet}

1) Formule et notation de base: On considère la suite finie $\left(x_{n}\right)$ des $N$ éléments correspondant à un signal $x$ échantillonné et limité par une fenêtre rectangulaire. La TFD de $x$ correspond à la suite finie $\left(X_{m}\right)$ des $N$ éléments obtenus par la somme finie et discrète

$$
X(m)=\sum_{n=0}^{N-1} x(n) e^{-2 i \pi \frac{m n}{N}},
$$

pour tout $m$ compris entre 0 et $N-1$. Ici, on choisit comme souvent le nombre d'échantillons de la TFD égal à celui du signal. Dans la suite du texte, on notera $s$ le signal considéré car la notation $x$, associée aux coordonnées $y$ et $z$ (Eq. (5)), sera utilisée pour la représentation 3D elle-même.

S'inspirant de l'usage le plus courant, on note les exponentielles complexes :

$$
W_{N}^{m n}=e^{-2 i \pi \frac{m n}{N}} .
$$

Parmi toute la littérature intéressante disponible sur le traitement du signal, on renvoie le lecteur aux références [1-3] pour une présentation de la TFD sous ses divers aspects.

2) Intérêt du projet: Quel intérêt notre approche présentet-elle, pour les étudiants et pour nous-mêmes, et en quoi se distingue-t-elle de celles que l'on rencontre habituellement? Il existe beaucoup de chapitres de manuel, de polycopiés et de cours de présentation disponibles sur la TFD. Certaines de ces présentations sont approfondies, de grande qualité et sont soutenues par des illustrations graphiques très explicites sur tel ou tel point. Ceci étant, elles reposent toujours sur les représentations temporelles, fréquentielles ou temps-fréquence usuelles. Ici, nous voulons faire l'examen des objets présents dans la construction de la TFD en tant que tels, de sorte à en comprendre la structure avant de considérer la TFD par elle-même. De plus, la présentation de la TFD telle qu'elle est faite ici constitue un ensemble de «thèmes tiroirs » qu'il est possible d'ouvrir et d'approfondir autant que désiré en fonction de la curiosité, du temps disponible et des compétences des étudiants et des enseignants : nombres complexes (représentations géométrique et vectorielle, racines $n$-ièmes de l'unité), arithmétique (divisibilité, congruence), groupes finis, visualisation $2 \mathrm{D}$ et $3 \mathrm{D}$, utilisation de divers logiciels (Matlab®), Scilabß), Geogebraß).

\section{Rôle des entiers $n, m$ et $N$}

La première difficulté rencontrée par les étudiants concerne le rôle des entiers présents dans les expressions (1) et (2). Dans le compte-rendu de leur projet (en début de soutenance, en 
particulier), les étudiants doivent présenter très clairement le rôle joué par chacun d'entre eux. Sans cela, l'exposé tout entier reste obscur et les figures tracées dans la suite, quasiment illisibles.

- L'entier $n$ : il est associé à la discrétisation du temps selon la relation $t_{n}=n T_{e}$, où $T_{e}$ est la période d'échantillonnage du signal. On convient que $n$ varie de 0 à $N-1$.

- L'entier $m$ : il correspond à la discrétisation de la fréquence par «pas » de $\Delta f$. On écrit donc

$$
f_{m}=m \Delta f \text {. }
$$

Si le pas en fréquence est $\Delta f=F_{e} / N$, l'axe des fréquences est alors gradué tous les $1 /\left(t_{b}-t_{a}\right)$, où $t_{b}-t_{a}$ est la largeur de la fenêtre encadrant le signal. En effet,

$$
\frac{F_{e}}{N}=\frac{1}{T_{e} N}=\frac{1}{t_{b}-t_{a}} \text {. }
$$

- L'entier $N$ : il représente le nombre d'échantillons, tant temporels que fréquentiels. La problématique du calcul rapide par FFT conduit à prendre $N$ comme une puissance de 2. Pour rendre notre étude très simple et visuellement bien explicite, on se limitera, sauf mention contraire, à $N=2^{3}=8$.

\section{REPRÉSENTATION 3D DES TERMES $W_{N}^{m n}$}

\section{A. Des escaliers en colimaçon}

Selon la formule (1), chaque terme de la suite associée à la TFD correspond à une valeur de $m$. Les entiers $N$ et $m$ étant fixés, on va donc visualiser l'évolution des $W_{N}^{m n}$ en fonction de l'entier $n$. L'idée est alors d'attribuer à un point de l'espace les coordonnées $x, y$ et $z$ associées aux $W_{N}^{m n}$ en posant, pour $n$ compris entre 0 et $N-1$ [4-5] :

$$
\begin{aligned}
x(n) & =\cos (2 \pi m n / N), \\
y(n) & =-\sin (2 \pi m n / N) \\
\text { et } z(n) & =n .
\end{aligned}
$$

Dans le cas où $N=8$, on obtient les huit graphes Fig.1 que l'on appellera « escaliers » dans la suite du texte. À une valeur de $m$ correspond donc un escalier dont les « marches », en bleu, tournent autour d'un axe central, en rouge. Elles tournent dans le sens indirect (sens des aiguilles d'une montre) jusqu'à $m=N / 2$, dans le sens direct, au-delà. Chaque marche correspond à une valeur de $n$ et les points de coordonnées $x(n), y(n), z(n)$ (à l'extrémité de chaque marche) sont reliés par un segment vert. Pour une valeur de $N$ et pour chaque valeur de $m$, on a donc la correspondance

Une marche :

$$
W_{N}^{m n}
$$

Un escalier :

$$
E_{N}^{m}:=\left\{1, W_{N}^{m 1}, W_{N}^{m 2}, \ldots, W_{N}^{m(N-1)}\right\}
$$

Sur chaque graphe Fig.1, on lit

- la valeur $F_{e}=1 / T_{e}$ de la fréquence d'échantillonnage. Ici, avec $T_{e}=\left(t_{b}-t_{a}\right) / N=20 / 8, F_{e}=0,4$ en unités arbitraires.

- la valeur de $f_{m}$ donnée par (3) :

$$
f_{m}=m \frac{F_{e}}{N}=m \times \frac{0,4}{8}=m \times 0,05 .
$$

- les valeurs $X_{x}$ et $X_{y}$ des sommes sur $n$ de $x(n)$ et $y(n)$ :

$$
X_{x}=\sum_{n=0}^{N-1} x(n) \quad \text { et } \quad X_{y}=\sum_{n=0}^{N-1} y(n) .
$$

- la valeur notée $A b s(X)$ correspond au module :

$$
\operatorname{Abs}(X)=\left|X_{x}+i X_{y}\right|=\left|\sum_{n=0}^{N-1} W_{N}^{m n}\right| .
$$

C'est donc aussi le module de la somme vectorielle de tous les « vecteurs-marches » (projetés dans le plan $x O y$ ). Hormis le premier escalier $E_{8}^{0}(m=0$ et $N=8)$, toutes les valeurs de $A b s(X)$ sont nulles, conformément à l'observation des orientations de toutes les marches qui balayent uniformément les $2 \pi$ radians.
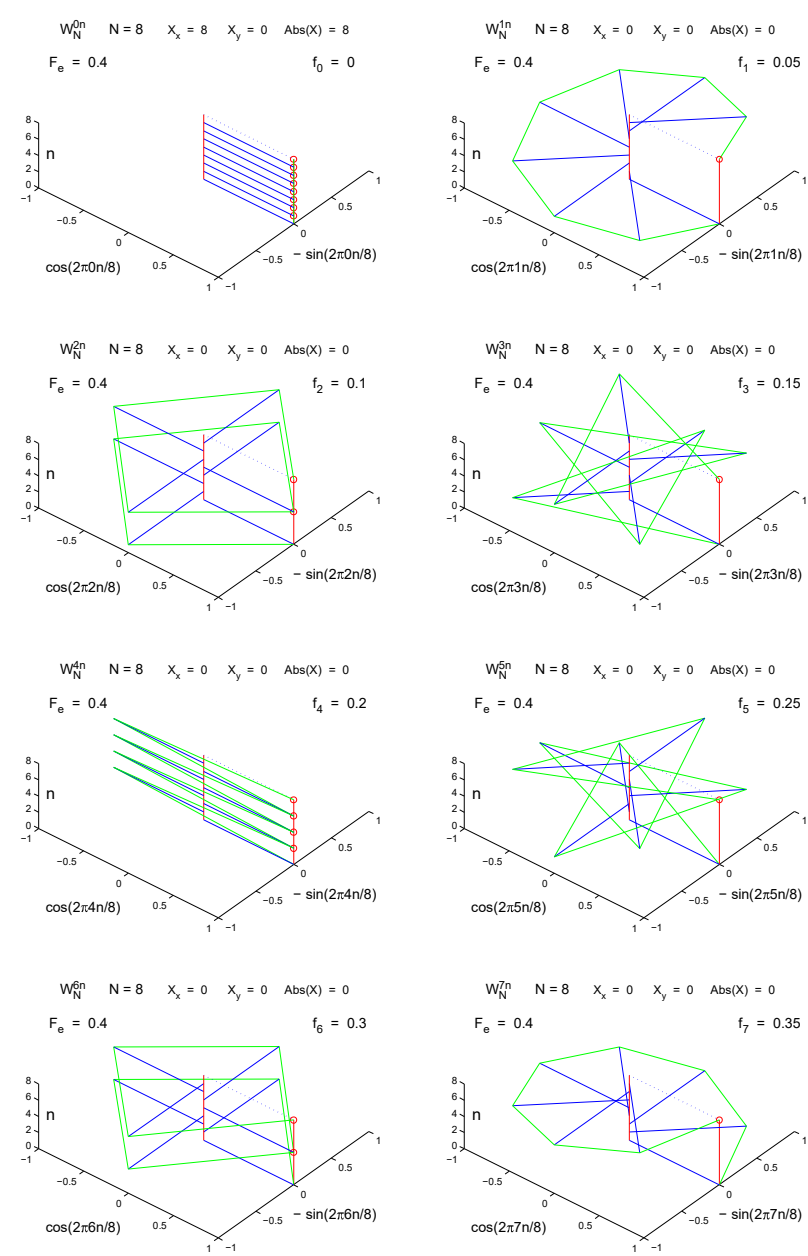

FIGURE 1: Représentation 3D des $W_{N}^{m n}$ de $m=0$ à $m=7$

\section{B. Ce que l'on voit dans et par les escaliers}

1) Périodicités: Après la symétrie sur les indices $m$ et $n$, $\left(W_{N}^{m n}=W_{N}^{n m}\right)$, la propriété la plus évidente des $W_{N}^{m n}$ est leur périodicité. Toujours de module $1, W_{N}^{m n}$ retrouve une même valeur complexe pour certaines valeurs de $m$ et de $n$ : quel que soit $k$ entier,

$$
W_{N}^{m(n+k N)}=W_{N}^{m n} \quad \text { et } \quad W_{N}^{(m+k N) n}=W_{N}^{m n} .
$$


2) Symétrie entre escaliers: On observe que les escaliers peuvent s'associer par paire. En effet, les escaliers $E_{8}^{1}(m=$ $1, N=8$ ) et $E_{8}^{7}, E_{8}^{2}$ et $E_{8}^{6}, E_{8}^{3}$ et $E_{8}^{5}$ se déduisent l'un de l'autre par symétrie par rapport au plan $x O z:$ l'escalier $E_{8}^{7}$ est identique au $E_{8}^{1}$, mais tourne dans le sens contraire. De même pour les escaliers $E_{8}^{2}$ et $E_{8}^{6}, E_{8}^{3}$ et $E_{8}^{5}$. L'escalier $E_{8}^{4}$ est alors son propre symétrique. Ceci correspond à la propriété :

$$
W_{N}^{(N-m) n}=\left(W_{N}^{m n}\right)^{*}
$$

3) Symétrie dans chaque escalier: A l'intérieur d'un même escalier, certaines marches sont symétriques par rapport à l'axe central. Par exemple, pour $m=1$, les marches $n=0$ et 4 , $n=1$ et 5 , etc., sont en opposition. Pour $m=2$, ce sont les marches $n=0$ et $2, n=1$ et 3 , etc. Dans tous les cas, on cherche l'entier relatif $q$ tel que

$-2 \pi m(n+q) / N=-2 \pi m n / N+\pi+2 k \pi, \quad k$ entier.

On a donc, pour $N=8$

$$
q_{k}=-N(2 k+1) /(2 m)=-4(2 k+1) / m .
$$

Pour $m=1, q_{0}=-4$; pour $m=2, q_{0}=-2$; pour $m=3$, $q_{1}=-4$; pour $m=4, q_{0}=-1$. C'est bien le nombre de marches à ajouter, comptées dans le sens indirect (Fig. 1).

4) Tours exacts avant $n=N$ et $P G C D$ de $m$ et $N$ : De façon moins grossière qu'en 1) $W_{N}^{m n}$ peut reprendre une valeur réelle ou complexe identique, par exemple $W_{N}^{m n}=1$, avant que $n$ ne prenne la valeur $N$. En particulier, on observe, quand ils se réalisent, l'existence de tours « exacts » effectués par les $W_{N}^{m n}$ avant que $n=N$. Sur les escaliers $E_{N}^{m}$, on peut aussi compter ces tours.

Sur les graphes Fig. 1, on constate que ceci a lieu pour certains escaliers, et pas pour d'autres. Pour $m=0,2,4$ et 6 , on observe en effet que certains points se trouvent à l'aplomb les uns des autres avant que $n=N=8$ (trait rouge vertical en $(1,0)$ ). Par exemple, pour $m=2$, on constate que :

$$
W_{8}^{24}=W_{8}^{20}, \quad W_{8}^{25}=W_{8}^{21} \quad \text { et } \quad W_{8}^{26}=W_{8}^{22} .
$$

Pour $m=4$, la cyclicité est plus grande et les égalités plus nombreuses pour un même point. Si $N$ est une puissance de 2 , on vérifie, pour $m \neq 0$, qu'elles sont en nombre maximal pour $m=N / 2$.

Dans les premiers membres des égalités (15), $n$ représente le nombre de marches au bout desquelles la valeur du membre de droite est atteinte. La différence entre les deux valeurs de $n$ donne le nombre de marches contenues dans un tour exact, ici 4. Pour la première égalité, l'entier $n$ doit satisfaire, pour $m$ et $N$ fixés :

$$
e^{-2 i \pi \frac{m n}{N}}=1 \quad \text { et } \quad 0<n<N .
$$

- Si $m$ et $N$ sont premiers entre eux, seul $n=N$ vérifie $e^{-2 i \pi \frac{m n}{N}}=1$ et aucun point (avant $n=N$ ) ne se trouve au dessus d'un autre. Aucun « tour »n'est alors achevé ou « exact»: les escaliers $E_{8}^{1}$ et $E_{8}^{3}$ (idem pour $E_{8}^{5}$ et $E_{8}^{7}$ ) illustrent clairement ce point. Un tour complet (de $n=0$ à $n=N)$ correspond alors, dans le plan $x O y$, à un polygone régulier à $N$ sommets.
- Si $m$ et $N$ ne sont pas premiers entre eux et ont pour PGCD $g>1, m / N=m^{\prime} / N^{\prime}$ où $m=g m^{\prime}$ et $N=g N^{\prime}$. Le nombre de marches à « monter » avant d'atteindre la valeur 1 (vérifiant (16)) est donné par

$$
\left\{\begin{array}{l}
n_{k}=k N / m=k N^{\prime} / m^{\prime}, \quad k \in \mathbb{N} . \\
0<n_{k}<N
\end{array} .\right.
$$

Ayant $n_{k}$ entier, on a $k=m^{\prime}$ et $n_{k}=N^{\prime}=N / g$. Un tour exact correspond donc, dans le plan $x O y$, à un polygone régulier à $N / g$ sommets. D'autre part, le nombre de tours exacts $n_{t}$ effectués entre $n=0$ et $n=N$ est simplement $n_{t}=N / n_{k}=g=\operatorname{PGCD}(m, N)$. C'est bien ce qu'on vérifie dans les escaliers $E_{8}^{2}, E_{8}^{4}$ et $E_{8}^{6}$ Fig. 1 . Dans le cas où $m$ et $N$ sont premiers entre eux, on a aussi, évidemment, $n_{t}=1=\operatorname{PGCD}(m, N)$.

On donne Fig. 2 quelques exemples d'escaliers où l'on distingue clairement, pour des valeurs de $m$ et de $N$ intéressantes, le nombre de marches à l'aplomb de $(1,0)$ et le nombre de tours (les ronds rouges) effectués.
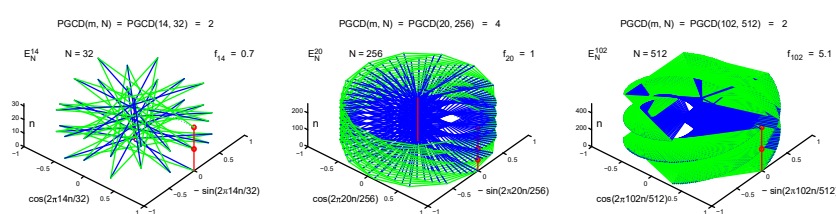

FIgURE 2: Exemples de «tours exacts » et PGCD de $m$ et $N$

5) Une autre symétrie entre escaliers : Enfin, une observation attentive des escaliers conduit à relier ceux dont les valeurs de $m$ diffèrent de $N / 2$. En effet, on vérifie aisément que

$$
W_{N}^{(m+N / 2) n}=(-1)^{n} W_{N}^{m n} .
$$

La mise côte-à-côte des escaliers Fig. 3 illustre clairement cette propriété pour $m=0$ et 4 , et de façon moins évidente pour $m=1$ et 5 . On examinera Fig. 1 les escaliers $E_{8}^{2}$ et $E_{8}^{6}$, et les escaliers $E_{8}^{3}$ et $E_{8}^{7}$.
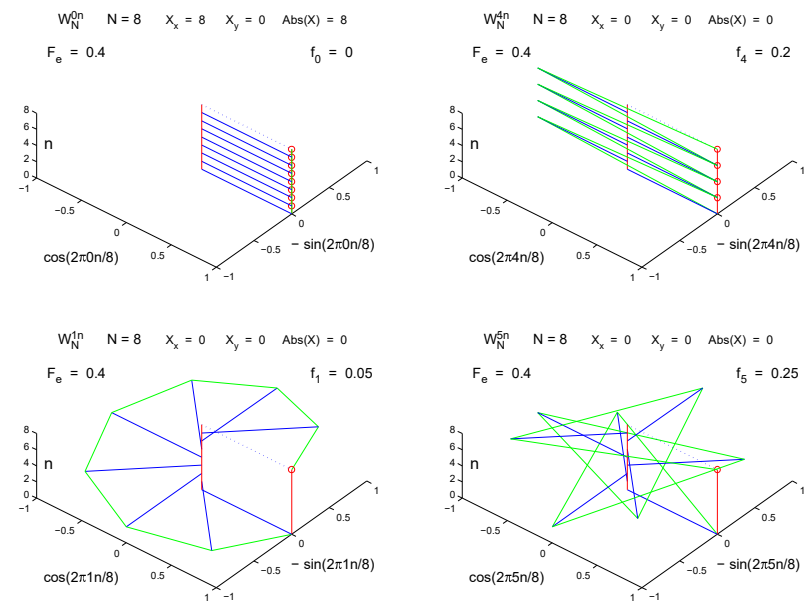

FIgure 3: Couples d'escaliers : $\left(E_{8}^{0}, E_{8}^{4}\right)$ et $\left(E_{8}^{1}, E_{8}^{5}\right)$ 


\section{GRANDES VALEURS DE $N$ : VERS LA CONTINUITÉ}

L'entier $N$ correspond au nombre d'échantillons du signal dans sa représentation temporelle comme dans sa représentation fréquentielle. Si $N$ augmente, les échantillons temporels « suivent » bien mieux la fonction continue associée au signal En ce qui concerne nos $N$ escaliers, leur nombre augmente. Au sein de chaque escalier, le nombre de marches augmente aussi. Elles définissent des secteurs angulaires de plus en plus fins et les segments verts périphériques qui les relient deviennent de plus en plus courts.

La figure 4 montre cette évolution qui conduit naturellement à des figures dont l'apparence est la continuité et la dérivabilité. Le quatrième graphique Fig. 4 correspond à une valeur de $N$ suffisamment grande pour sembler correspondre à une surface continue limitée par une spirale. On note qu'elle renferme néanmoins les mêmes propriétés que les escaliers correspondant à la même valeur de $m$ (§ II. B.) : nombre de tours avant que $n$ atteigne $N$, symétries, etc.
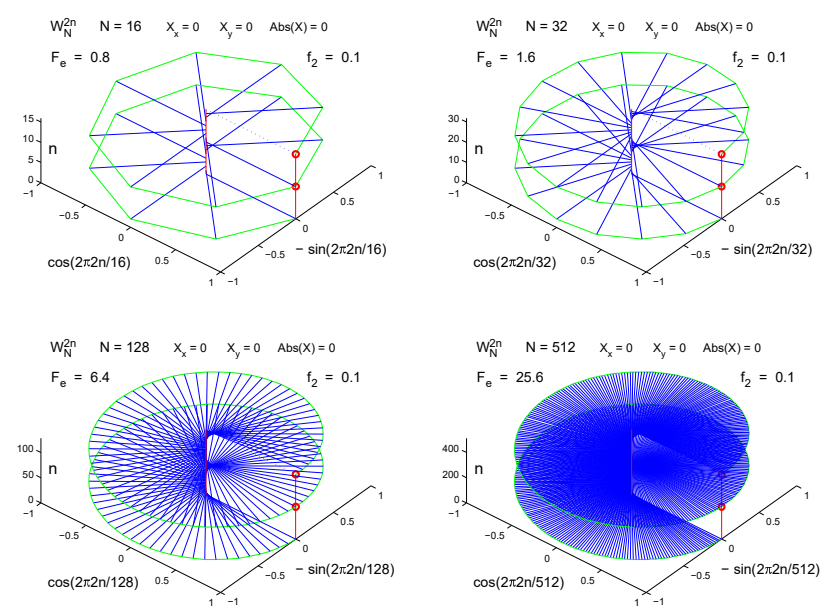

FIGURE 4: Escaliers pour $N$ croissant : $E_{16}^{2}, E_{32}^{2}, E_{128}^{2}$ et $E_{512}^{2}$

\section{GROUPE FINI ET MULTIPLICATION DES ESCALIERS}

Dans la section II., on donne une représentation 3D des exponentielles complexes $W_{N}^{m n}$. On montre facilement que, pour des valeurs de $N$ et de $n$ données, on a, quels que soient $m_{1}$ et $m_{2}$ :

$$
W_{N}^{\left(m_{1}+m_{2}\right) n}=W_{N}^{m_{1} n} W_{N}^{m_{2} n}
$$

Par conséquent, chaque produit de deux marches des escaliers $m_{1}$ et $m_{2}$ correspondant à une même valeur de $n$ est égal à la $n$-ième marche de l'escalier $m_{1}+m_{2}$.

Grâce à la périodicité sur la variable $m$ (propriété (11)), on montre aisément que tous les produits (19) conduisent à un coefficient $W_{N}^{\left(m_{1}+m_{2}\right) n}$ qui appartient à l'ensemble fini

$$
\left\{1, W_{N}^{1 n}, W_{N}^{2 n}, \ldots, W_{N}^{(N-1) n}\right\} .
$$

L' « escalier produit » est donc toujours un des huit escaliers Fig. 1 et la règle conduisant au produit de deux escaliers est

$$
\left\{\begin{array}{l}
E_{N}^{m_{1}} \star E_{N}^{m_{2}}=E_{N}^{m_{3}} \\
m_{3} \equiv\left(m_{1}+m_{2}\right) \text { modulo } N \\
0 \leq m_{3}<N-1
\end{array} .\right.
$$

Les deux exemples de la figure 5 illustrent la règle (21) : $E_{8}^{1} \star E_{8}^{2}=E_{8}^{3}$ et $E_{8}^{2} \star E_{8}^{7}=E_{8}^{1}$. Pour la $n$-ième marche $W_{N}^{m_{3} n}$ de l'escalier produit $E_{N}^{m_{3}}$, on observera la somme des angles associés aux $n$-ièmes marches $W_{N}^{m_{1} n}$ et $W_{N}^{m_{2} n}$.

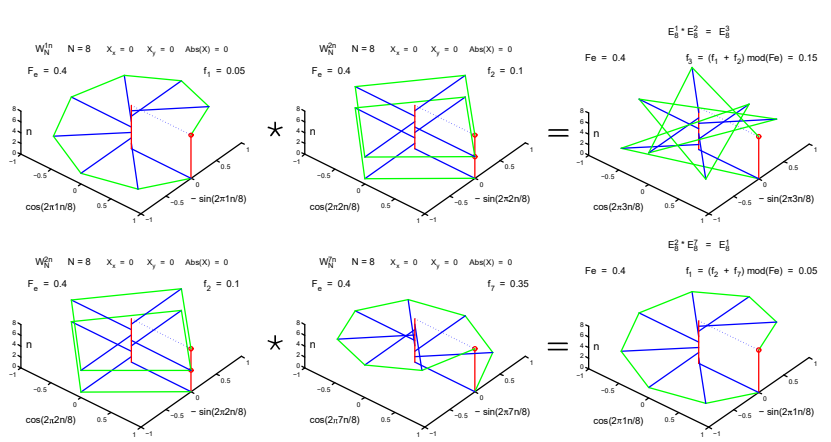

FIGURE 5: Les produits d'escaliers $E_{8}^{1} \star E_{8}^{2}$ et $E_{8}^{2} \star E_{8}^{7}$

La règle (21) et ses conditions conduisent à une table de multiplication des escaliers identique à la table d'addition modulo $N=8$ :

\begin{tabular}{|c|c|c|c|c|c|c|c|c|}
\hline$m_{1} \backslash m_{2}$ & 0 & 1 & 2 & 3 & 4 & 5 & 6 & 7 \\
\hline 0 & 0 & 1 & 2 & 3 & 4 & 5 & 6 & 7 \\
\hline 1 & 1 & 2 & 3 & 4 & 5 & 6 & 7 & 0 \\
\hline 2 & 2 & 3 & 4 & 5 & 6 & 7 & 0 & 1 \\
\hline 3 & 3 & 4 & 5 & 6 & 7 & 0 & 1 & 2 \\
\hline 4 & 4 & 5 & 6 & 7 & 0 & 1 & 2 & 3 \\
\hline 5 & 5 & 6 & 7 & 0 & 1 & 2 & 3 & 4 \\
\hline 6 & 6 & 7 & 0 & 1 & 2 & 3 & 4 & 5 \\
\hline 7 & 7 & 0 & 1 & 2 & 3 & 4 & 5 & 6 \\
\hline
\end{tabular}

TABLE I: Composition des escaliers

\section{REPRÉSENTATION 3D DES PRODUITS $s(n) W_{N}^{m n}$}

\section{A. Ce que l'on veut faire}

On désire à présent effectuer le même travail que précédemment, mais en présence d'un signal $s$ échantillonné et fenêtré. Ses $N$ échantillons sont alors multipliés aux coefficients $W_{N}^{m n}$ selon la formule (1). Il est clair que, suite à ces multiplications, les escaliers de la Fig. 1 seront affectés dans leur forme et leur symétrie. Pour visualiser aisément ces modifications, nous choisissons le signal le plus simple, une sinusoïde $s$ caractérisée par une seule fréquence $f_{s}=0,1$ en unité arbitraire :

$$
t \mapsto s(t)=\sin \left(2 \pi f_{s} t\right)
$$

\section{B. Tracés des TFD d'un signal sinusoïdal}

À titre de rappel, nous traçons Fig. 6a le sinus $s$ (22) limité et échantillonné, puis Fig. 6b son spectre d'amplitude obtenu par la formule (1) puis par la commande « $\mathrm{fft} »$ sous Matlabß. 
Les deux tracés Fig. 6 b coïncident et font tous deux apparaître la symétrie propre à la TFD soulignée $\S$ II. B.2) et visualisée Fig. 1 : la formule (12) implique que, pour $s$ réel

$$
|X(N-m)|=|X(m)| .
$$

Une raie apparaît bien en $f=f_{s}$, mais aussi en $f=F_{e}-f_{s}$, où, ici, la fréquence d'échantillonnage du signal vaut $F_{e}=$ $1 / T_{e}=0,4$ en unité arbitraire.

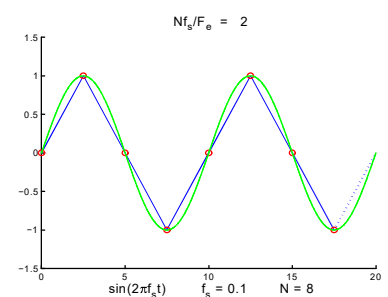

(a) Sinus échantillonné

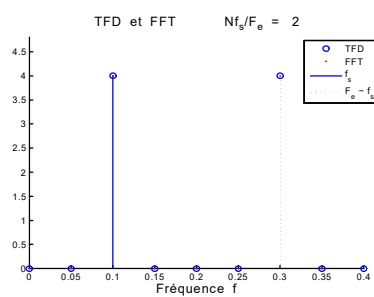

(b) Spectre d'amplitude
FIGURE 6: Graphe de $\sin \left(2 \pi f_{s} t\right)$ échantillonné et son spectre d'amplitude pour $f_{s}=0,1=2 F_{e} / N$

N. B. : Dans les graphes des différentes TFD, on décide d'indiquer leur $N+1$-ième valeur (le dernier rond bleu où $m=$ $N)$. On souligne ainsi la périodicité de la TFD et, pour $s$ réel, la symétrie des spectres d'amplitude par rapport à $m=N / 2$.

\section{C. Être ou ne pas être multiple de $F_{e} / N$}

Dans l'étude et l'observation d'une fréquence $f_{s}$ incluse dans le spectre d'un signal, il est crucial de bien distinguer deux cas visuellement très différents. Si $f_{s}$ est multiple de $F_{e} / N$, alors $f_{s}$ se situe exactement sur une graduation de l'axe des fréquences discrétisées selon (3) et (4). Dans ce cas, un pic indique exactement la valeur de $f_{s}$, les amplitudes voisines étant alors exactement nulles (Fig. 6b et Fig. 7a).

Dans le cas contraire, les points se répartissent sur le motif spectral de la fenêtre, centré en $f_{s}$. Ici, aucun des pics du spectre n'indique exactement la valeur de $f_{s}$ et de l'amplitude associée. C'est ce que montre le graphe Fig. $7 b$.

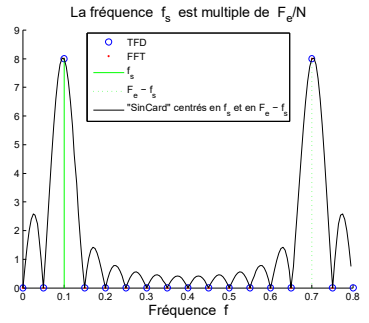

(a) $f_{s}$ est multiple de $F_{e} / N$

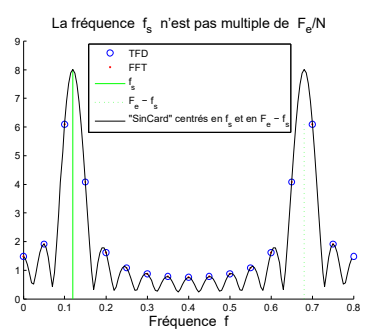

(b) $f_{s}$ n'est pas multiple de $F_{e} / N$
FIGURE 7: $f_{s}$ est ou n'est pas multiple de $F_{e} / N$

\section{Déformation des escaliers par le signal $s$}

1) Construction des nouveaux escaliers : Les escaliers avec signal doivent nous montrer, pour un nombre d'échantillons $N$ fixé et pour chaque valeur de $m$, l'évolution des produits $s(n) W_{N}^{m n}$ en fonction de l'entier $n$. Les coordonnées $x$ et $y$ données par les relations (5) deviennent alors

$$
\begin{aligned}
x(n) & =s(n) \cos (2 \pi m n / N), \\
y(n) & =-s(n) \sin (2 \pi m n / N) \\
\text { et } z(n) & =n .
\end{aligned}
$$

Pour $s$ donné par (22) (Fig. 6a), on obtient les escaliers Fig. 8. Si les axes des nouveaux escaliers sont définis par (24), on continue, pour alléger la notation dans les graphes, à noter les axes $x$ et $y$ comme précédemment.

Les grandeurs $X_{x}, X_{y}$ et $A b s(X)$ indiquées dans chaque graphe sont définies de la même façon qu'en (9) et en (10), mais avec les nouvelles expressions données par (24). Les marches et escaliers définis par (6) et (7) s'écrivent à présent

Une marche : $\quad s(n) W_{N}^{m n}$

Un escalier : $E_{N}^{m}(s):=\left\{s(n) W_{N}^{m n}, n=0, \ldots, N-1\right\}(26)$

2) Ce que l'on observe; l'amplitude et la phase de la TFD: Les escaliers Fig. 8 présentent des différences mais aussi des points communs avec ceux de la Fig. 1. On observe toujours certaines des propriétés indiquées $§$ II. $B$..
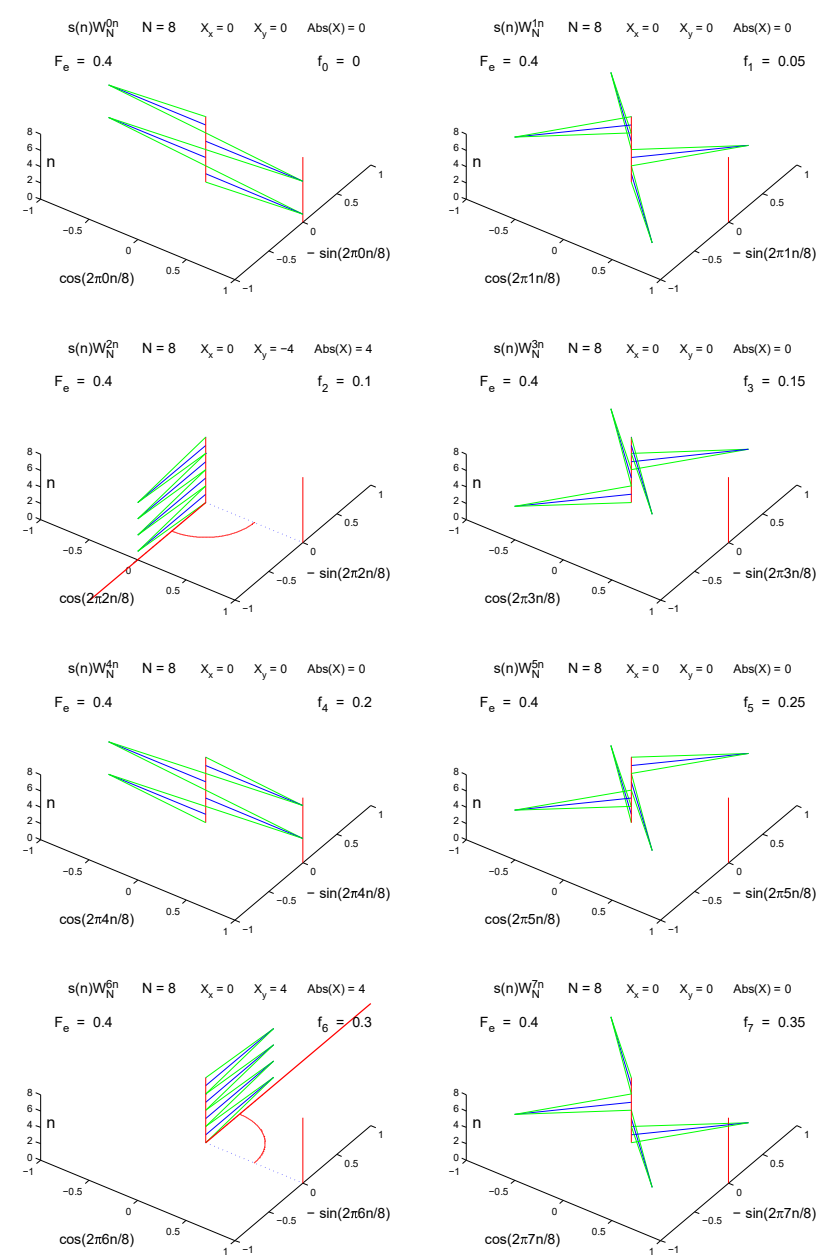

FIGURE 8: Escaliers pour $s(t)=\sin \left(2 \pi f_{s} t\right)$ où $f_{s}=0,1$ 
Indépendamment du signal $s$ choisi, sont préservées la périodicité modulo $N$ (formules (11)) et, pour $s$ réel, la symétrie observée lors de la substitution $m \rightarrow N-m$ (formule (12)). Par contre, pour chaque escalier, l'évolution des marches (les segments bleus) en fonction de $n$ est perturbée par la présence du signal $s$. Pour les escaliers correspondant aux valeurs de $m$ égales à $0,1,3,4,5$ et 7 , on voit clairement que la somme vectorielle des vecteurs associés aux marches est exactement nulle, ce qu'indiquent les calculs dans chaque graphe $(\operatorname{Abs}(X)=0)$.

La fréquence $f_{s}$ étant proportionnelle à $\Delta f=F_{e} / N$ (cf. $\S \mathrm{V}$. $C$. et $\S$ VI.), il existe un $m$ tel que l'on ait exactement

$$
f_{s}=f_{m} \text {. }
$$

En effet, pour $m=2$ et $m=6$, on observe Fig. 8 que cette somme vectorielle est non nulle. Les valeurs de l'entier $m$ associées à ces escaliers correspondent aux fréquences données par $f_{m}=m F_{e} / N$ et $F_{e}-f_{m}$, soit 0,1 et 0,3 en unité arbitraire. Ce sont bien les fréquences $f_{s}$ et $F_{e}-f_{s}$ attendues. Réciproquement, on obtient facilement la première valeur de $m$ cherchée par

$$
m_{s}=\frac{f_{s}}{\Delta f}=\frac{f_{s} N}{F_{e}}=\frac{0,1 \times 8}{0,4}=2 .
$$

Le module indiqué de la somme vectorielle des marches est alors 4 , valeur de l'amplitude des pics associés aux fréquences $f_{s}$ et $F_{e}-f_{s}$ dans le spectre Fig. 6b. En effet, un calcul élémentaire effectué à l'aide de (1) conduit, pour $m=2$ et $m=6$, à

$$
X(2)=-4 i \quad \text { et } \quad X(8-2)=X(6)=4 i .
$$

Sur les escaliers $E_{8}^{2}(s)$ et $E_{8}^{6}(s)$ Fig. 8, la somme vectorielle de toutes les marches correspond au vecteur rouge dans le plan $x O y$. Ce vecteur somme donne deux indications :

- son module a pour valeur 4 comme indiqué dans le spectre d'amplitude Fig. $6 \mathrm{~b}$ aux fréquences $f_{s}$ et $F_{e}-f_{s}$;

- sa phase est $-\pi / 2$ pour $m=2$ et $\pi / 2$ pour $m=6$ pour ces mêmes fréquences, en accord avec (29).

Un examen plus approfondi de la phase dans notre représentation est donné § VIII..

3) Transformation des escaliers et sélection des fréquences : Pour le cas présent, très simple, où $N=8$ et $f_{s}=0,1$, il n'est pas difficile d'anticiper la façon dont les escaliers de la Fig. 1 vont se déformer en présence de $s$ (22) par les changements d'orientation des marches de chacun d'eux. Il suffit pour cela de multiplier les vecteurs associés aux huit marches par les huit valeurs que prend $s$ une fois échantillonné et fenêtré (cf. Fig. 6a) :

$$
\begin{gathered}
s(0)=0, \quad s(1)=1, \quad s(2)=0, \quad s(3)=-1, \\
s(4)=0, \quad s(5)=1, \quad s(6)=0, \quad s(7)=-1 .
\end{gathered}
$$

On compare Fig. 9 les cas $m=1$ et $m=2$ des Fig. 1 et 8 . La $n$-ième marche des escaliers de gauche se réoriente par la multiplication de l'échantillon $s(n)$. Au total, la somme vectorielle de toutes les marches conduit à une somme nulle pour les valeurs de $m$ ne correspondant pas à la fréquence $f_{s}$ et à une somme non nulle pour la valeur de $m$ associée à $f_{s}$.
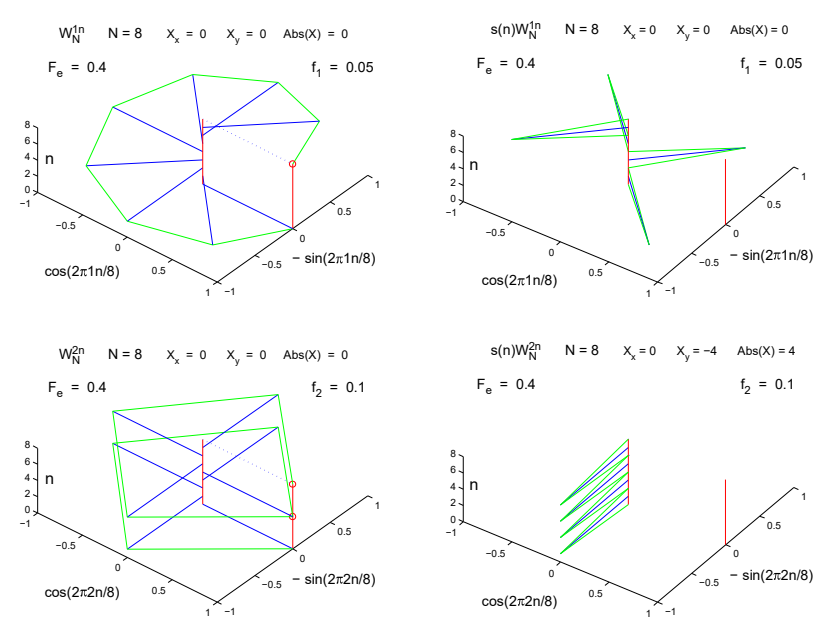

FIGURE 9: Transformation des escaliers $m=1$ et 2 pour un signal sinusoïdal de fréquence $f_{s}=f_{2}$

\section{Ne PAS ÊTRE MULTIPLE DE $F_{e} / N$}

Évoquons à présent le cas où la fréquence $f_{s}$ du signal $s$ (22) n'est pas multiple de $F_{e} / N$. Prenons $f_{s}=0,105$. Dans ce cas, comme on l'a déjà mentionné $\S \mathrm{V}$. C., aucun des points du spectre n'aura pour abscisse la valeur exacte de $f_{s}$. C'est bien ce que l'on observe Fig. 10 b.

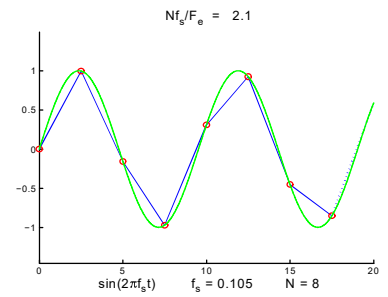

(a) Sinus échantillonné

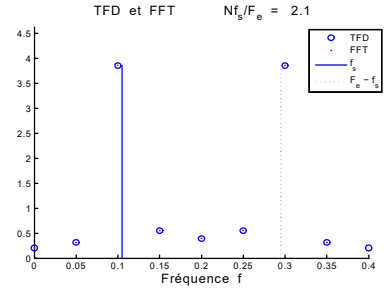

(b) Spectre d'amplitude
FIgURE 10: Sinus et sa TFD si $f_{s}$ n'est pas multiple de $F_{e} / N$

Si la relation (27) n'est donc pas satisfaite, il existe par contre une valeur de $m$ telle que

$$
f_{m}<f_{s}<f_{m+1} \text {. }
$$

Par conséquent, pour chaque valeur de $m$, la somme vectorielle sur les valeurs de $n$ est non nulle. Cependant, son module est plus important pour la valeur de $m$ correspondant à la fréquence approchant au mieux $f_{s}$. Comme on l'observe sur les escaliers Fig. 11, la répartition des marches est plus compliquée que dans la Fig. 8. Néanmoins, elle reste relativement lisible par rapport à la fréquence du signal : pour les valeurs de $m$ « ne correspondant pas » à $f_{s}$, les marches tournent autour de l'axe central de sorte que leur somme vectorielle soit presque nulle (cf. les valeurs de $A b s(X)$ ), alors que, pour $m=2$, on distingue clairement une orientation des marches dans un même sens. La somme vectorielle est alors maximale $(\operatorname{Abs}(X)=3,857<4)$. 

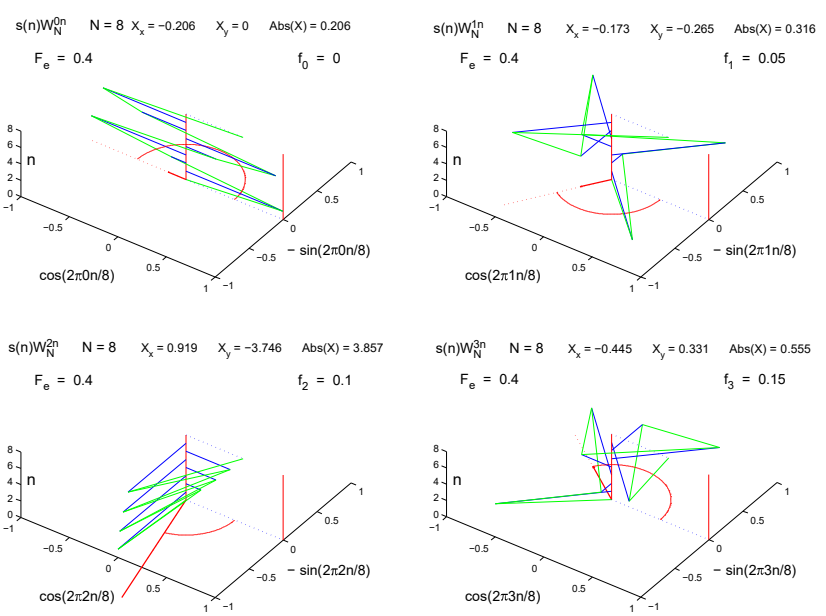

FIGURE 11: Quelques escaliers pour $f_{s}=0,105$

\section{VII. $N$ GRAND ET CONTINUITÉ}

Comme on l'a déjà mentionné dans la partie III., augmenter le nombre d'échantillons donne aux escaliers l'allure de surfaces «lisses » sans arêtes et limitées par une spirale. Elles gardent néanmoins les caractéristiques repérées dans leurs homologues associés à des valeurs de $N$ faibles (parties V. et VI.). On montre Fig. 12 quelques escaliers pour le signal sinusoïdal (22) pour les trois valeurs de $m: 1,2$ et 3 et les deux valeurs de $N: 16$ et 512 .
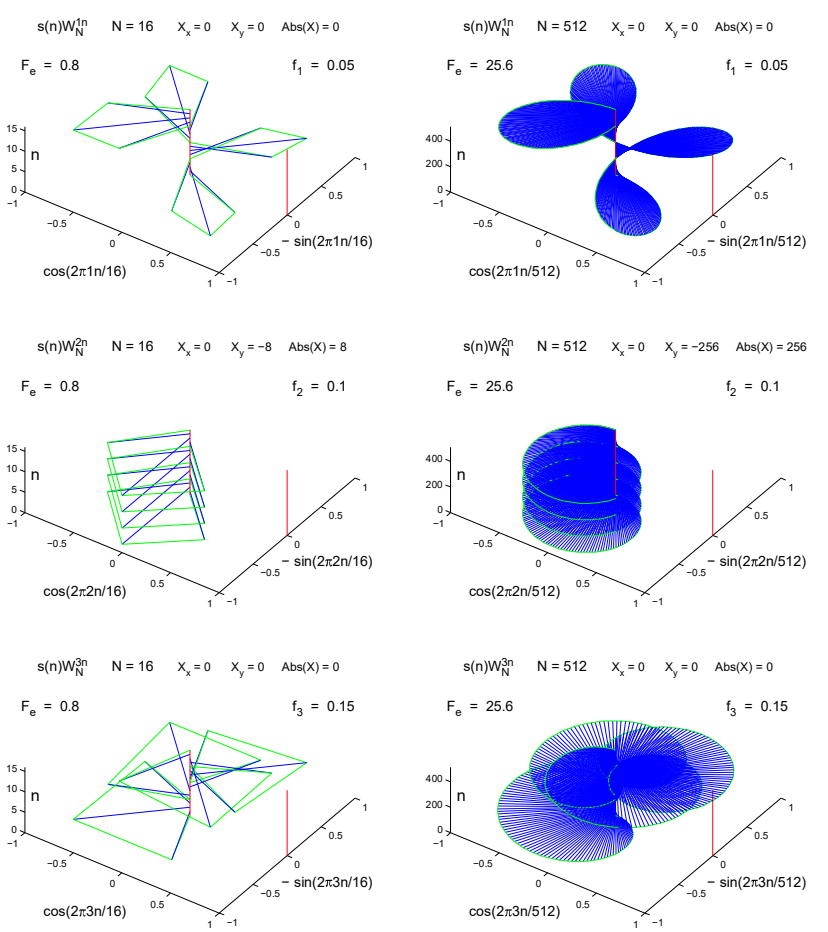

FIGURE 12: Escaliers avec signal $E_{16}^{1}(s)$ et $E_{512}^{1}(s), E_{16}^{2}(s)$ et $E_{512}^{2}(s), E_{16}^{3}(s)$ et $E_{512}^{3}(s)$

Comme attendu, si, pour une valeur de $m$ donnée, les escaliers changent fortement dans leur aspect, ils renferment bien les indications et propriétés requises : par exemple, la somme vectorielle des marches est bien nulle pour $f_{m} \neq f_{s}$ et non nulle pour $f_{m}=f_{s}=0,1=f_{2}$.

\section{VOIR LA « PHASE » EN 3D : LECTURE DE LA PHASE SUR LES ESCALIERS}

\section{A. Un escalier indique l'amplitude et la phase}

Mettons l'accent, dans cette partie, sur la lecture simultanée dans les escaliers de l'amplitude et de la phase associées à une fréquence. Pour la TFD comme pour la transformée de Fourier, l'accès visuel à l'information fréquentielle dans son intégralité nécessite l'examen de deux graphes : le spectre d'amplitude et le spectre de phase. Par construction, les graphes Fig. $6 \mathrm{~b}$ et Fig. 10b ne contiennent évidemment aucune information sur la « phase ». En particulier, ils ne donnent aucune information quant à la «situation temporelle absolue » du signal : le « départ » d'un sinus ou le centre d'une Porte, par exemple. A contrario, chaque « marche » de nos « escaliers » est associée à une longueur et une orientation. Par conséquent, l'examen d'un escalier nous donne, pour une fréquence donnée, les informations associées à l'amplitude et à la phase. C'est ce que l'on a vu $\S$ V. sur les escaliers $E_{8}^{2}(s)$ et $E_{8}^{6}(s)$ Fig. 8, pour le cas simple du signal sinusoïdal (22). Pour un échantillonnage bien meilleur (Fig. 12), par exemple pour $N=2^{9}=512$, on a

$$
X(2)=-256 i \quad \text { et } \quad X(512-2)=X(510)=256 i .
$$

Si l'amplitude a changé du fait d'un échantillonnage différent, la phase reste bien sûr la même $(-\pi / 2$ et $\pi / 2)$ comme on le constate sur les graphes du milieu Fig. 12.

\section{B. Cas général où $f_{s}$ n'est pas multiple de $F_{e} / N$}

Considérons le cas envisagé $\S$ VI. où la fréquence $f_{s}$ satisfait (31) en n'étant pas multiple de $F_{e} / N$. Si aucune des amplitudes n'est strictement nulle pour chacune des fréquences, aucune des phases (angles en rouge) n'est a priori nulle comme on le constate Fig. 11. Sur les graphes Fig. 13, on peut lire les spectres de phase du signal (22) pour les fréquences $f_{s}=0,1$ et $f_{s}=0,105$ considérées précédemment.

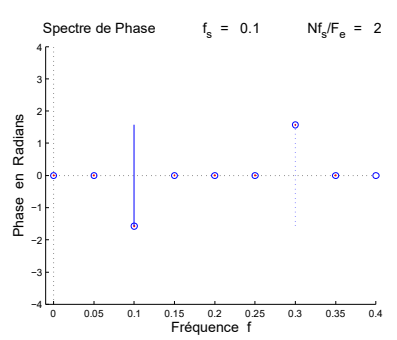

(a) $f_{s}$ multiple de $F_{e} / N$

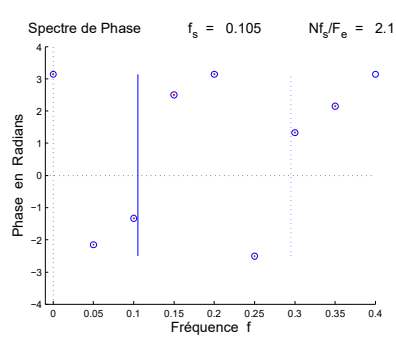

(b) $f_{s}$ non multiple de $F_{e} / N$
FIGURE 13: Spectre de phase

Dans les graphes Fig. 13a et Fig. 13b, on lit, pour chaque fréquence où l'amplitude est non nulle, la phase en radians indiquée dans l'escalier correspondant Fig. 8 et Fig. 11 : dans le premier cas, seules deux valeurs sont non nulles, dans le second, toutes les valeurs sont différentes de 0 . 


\section{Déphasage induit par un "retard»}

Considérons à présent le signal (22) $s$ « retardé » :

$$
t \mapsto s\left(t-t_{r}\right)=\sin \left(2 \pi f_{s}\left(t-t_{r}\right)\right), \text { où } \quad t_{r}>0 .
$$

On observe Fig. 14, pour deux valeurs de $N$ ( 8 et 512 ), les escaliers $E_{N}^{2}(s)$ pour trois valeurs de $t_{r}$. Sur ces graphes 3D, on peut lire l'angle dont les marches ont tourné à partir de leur orientation initiale dans $E_{N}^{2}(s)$ Fig. 8 ou Fig. 12 : angle entre les segments vert et rouge.

Si $t_{r}=p T_{e}, p \in \mathbb{N}$, chaque marche $W_{N}^{m n}$ est multipliée par $W_{N}^{m p}$. Tout l'escalier tourne alors (dans le sens indirect) d'un angle $\phi_{r}$ de $-2 \pi m p / N$ radians. Par exemple, pour $N=8$, $m=2$ et $t_{r}=2 T_{e}, \phi_{r}=-\pi$ (Fig. $14 \mathrm{~b}$ au centre).

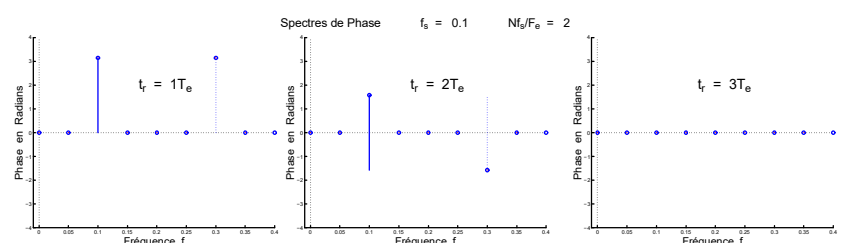

(a) Spectres de phase $(N=8)$ pour les retards $1 T_{e}, 2 T_{e}$ et $3 T_{e}$

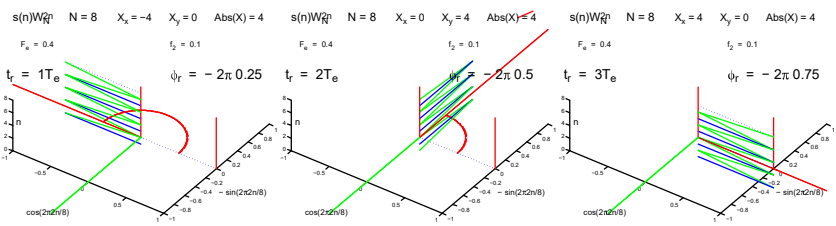

(b) Escaliers $E_{8}^{2}(s)$ pour les retards $1 T_{e}, 2 T_{e}$ et $3 T_{e}$

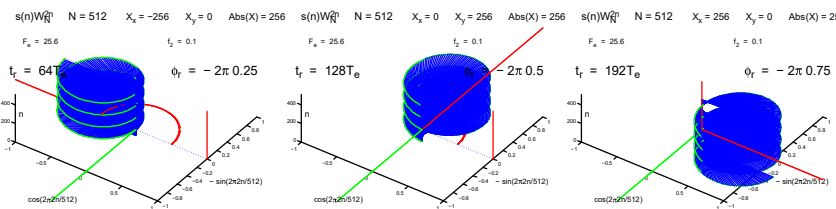

(c) Escaliers $E_{512}^{2}(s)$ pour les retards $64 T_{e}, 128 T_{e}$ et $192 T_{e}$

FIGURE 14: Rotation de l'escalier $E_{N}^{2}(s)$ suite à un retard

\section{SOMME}

Un signal quelconque est bien sûr plus complexe qu'un simple sinus et conduit à des TFD plus riches que celles envisagées ici. Dans cette trop courte introduction, nous ne pouvons pas faire l'analyse d'une représentation 3D de TFD plus complexes. Néanmoins, grâce aux décompositions de Fourier d'un signal, nous pouvons donner une idée de la façon dont les escaliers d'une TFD quelconque doivent se construire. Considérons un signal obtenu par une combinaison linéaire simple de deux sinus oscillant aux fréquences $f_{s_{1}}=0,05=$ $F_{e} / N$ et $f_{s_{2}}=0,15=3 F_{e} / N$ :

$$
s(t)=\sin \left(2 \pi f_{s_{1}} t\right)+\frac{2}{3} \sin \left(2 \pi f_{s_{1}} t\right)=s_{1}(t)+\frac{2}{3} s_{2}(t) .
$$

Les graphes Fig. 15 montrent le signal (34) et sa TFD en amplitude et en phase.

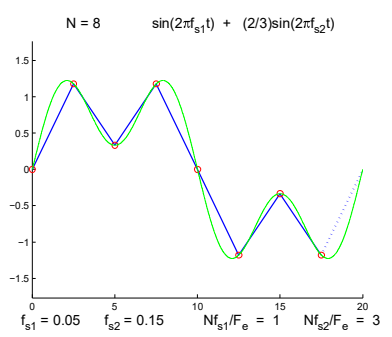

(a) Signal somme
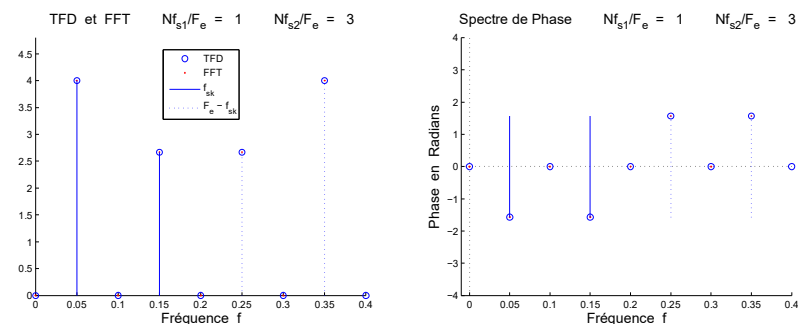

(b) Ses spectres d'amplitude et de phase

FIGURE 15: Un signal somme et sa TFD

Toujours à l'aide des formules (24), on construit les escaliers associés à notre représentation 3D de la TFD du signal somme (34). Les escaliers de $E_{8}^{0}(s)$ à $E_{8}^{5}(s)$ sont représentés Fig. 16. Comme on l'observe pour chacune des valeurs de $m$, amplitude et phase indiquées Fig. 15b sont données correctement par les escaliers.
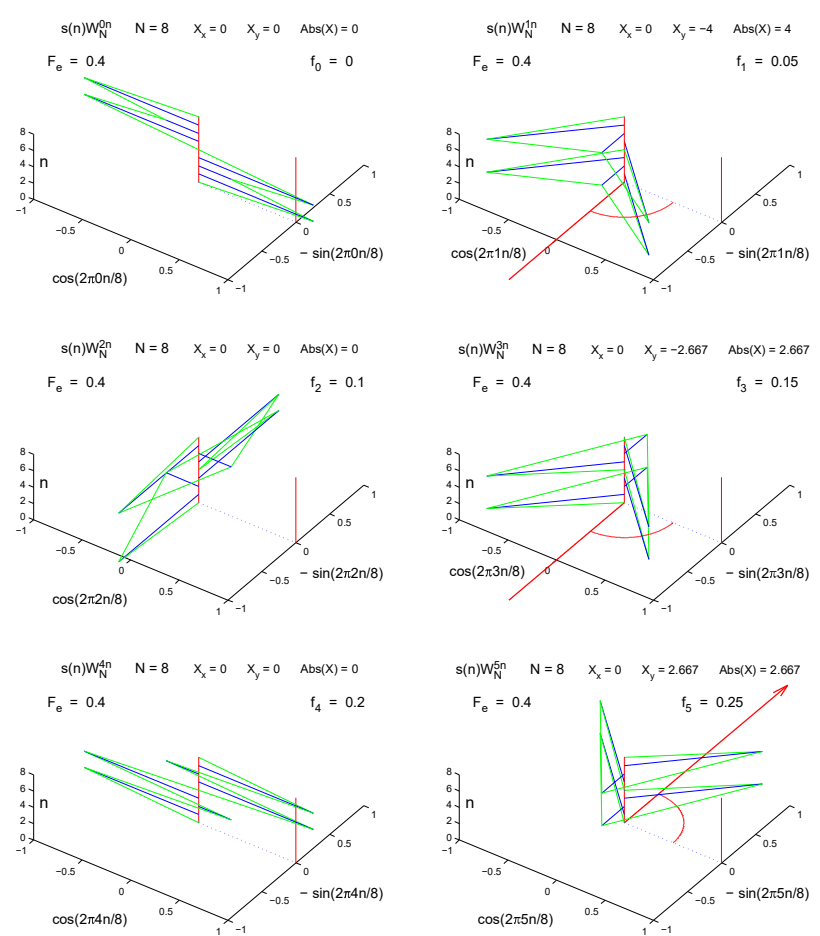

FIGURE 16: Escaliers pour un signal somme à 2 fréquences 0,05 et 0,15

Traduisant la linéarité de la TFD, chacun des escaliers 
Fig.16 s'obtient, pour un $m$ donné, par la somme pondérée des escaliers individuels associés aux deux fréquences $f_{s_{1}}=0,05$ et $f_{s_{2}}=0,15$. C'est ce que montrent les graphes Fig. 17 pour $m=1$ et $m=3$.

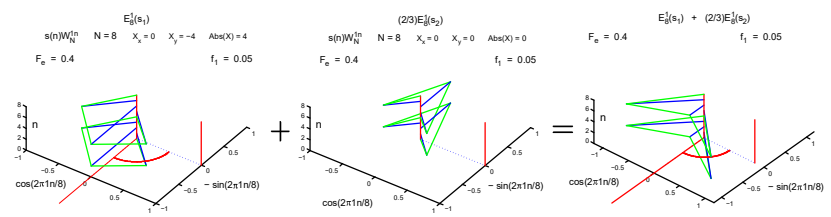

(a) Somme, pour $m=1$, des escaliers associés aux fréquences 0,05 et 0,15

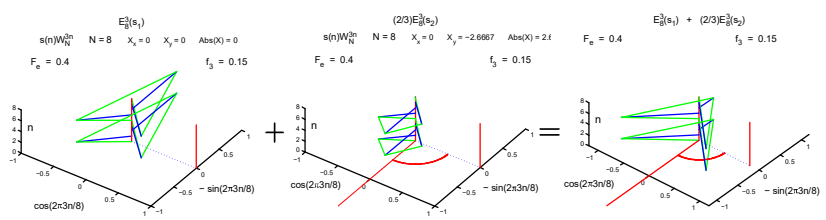

(b) Somme, pour $m=3$, des escaliers associés aux fréquences 0,05 et 0,15

Figure 17: Escaliers d'un signal somme et somme des escaliers

\section{VERS LA FFT}

L'algorithme conduisant à un calcul « rapide » de la TFD repose sur certaines des propriétés des coefficients $W_{N}^{m n}$. En particulier, un calcul élémentaire montre que

$$
W_{N}^{2 m n}=W_{N / 2}^{m n} \text {. }
$$

Sans pouvoir approfondir ici ce qui constitue un sujet en soi, indiquons juste que certaines séquences dans la somme (1) se reproduisent alors à l'identique, ce qui conduit à une réduction possible du nombre d'opérations associées au calcul de (1). En tenant compte de $0 \leq n<N$ les escaliers $E_{N}^{m}$ Fig. 18 illustrent les égalités et inclusions

$$
W_{32}^{8 n}=W_{16}^{4 n}=W_{8}^{2 n} \quad \text { et } \quad E_{8}^{2} \subset E_{16}^{4} \subset E_{32}^{8} .
$$
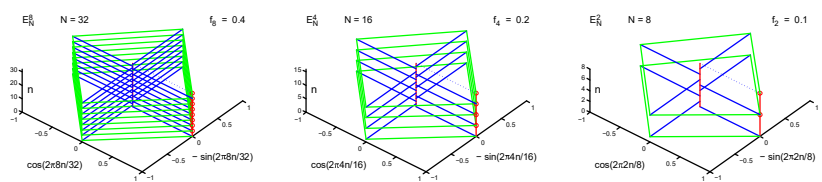

FIGURE 18: Escaliers « congruents » pour $N=32,16,8$

\section{Conclusion}

\section{A. Conclusion et perspectives}

Les mathématiques invoquées dans ce travail ne sont pas particulièrement difficiles et devraient être bien maîtrisées par un étudiant engagé dans des études supérieures technicoscientifiques : exponentielles complexes et formule d'Euler, sommes discrètes finies, sommes vectorielles et représentation de Fresnel, symétries spatiales, éléments de théorie des groupes.

Ces simples notions nous ont permis, d'une part, de clairement visualiser les propriétés et caractéristiques de la TFD dans des figures 3D - les « escaliers » - et, d'autre part, de lire, pour une fréquence donnée et un signal donné, amplitude et phase au sein d'un même escalier.

Pour une étude ultérieure, trois sujets au moins méritent une attention plus poussée. Premièrement, l'étude dans notre représentation 3D de la TFD de signaux plus riches que les simples oscillations utilisées ici. Deuxièmement, la même étude de signaux simples ou complexes, mais très finement échantillonnés, i.e. pour de grandes valeurs de $N$ (Cf. Fig. 4 et 12). Enfin, il sera intéressant de relier précisément les symétries décrites $\S$ II. aux procédures de calcul rapide de la TFD, en particulier à celle associée à la FFT usuelle.

\section{B. Bilan pédagogique}

L'expérience montre que les étudiants ont bien du mal à utiliser le bagage élémentaire rappelé ci-dessus pour mettre en évidence ce que l'on voit par les graphes 3D et les relier aux caractéristiques de la TFD énumérées dans une présentation classique. En particulier, les étudiants peuvent avoir des difficultés à simplement compter tel ou tel élément au sein d'une figure donnée : nombre de marches dans un escalier ayant telle propriété, nombre de tours effectués avant $n=N$, etc. Le travail présenté ici nécessite donc, d'une part, de se replonger dans l'utilisation des différents sujets mentionnés ci-dessus et, d'autre part, un examen très soigné de ce que renferme la TFD. Sans cela, la représentation décrite ici reste totalement obscure et son intérêt n'apparaît absolument pas. De plus, il faut faire preuve d'un minimum d'initiatives pour utiliser ce qu'offrent les logiciels de calcul numérique Matlabß et Scilabß. Par conséquent, seuls des groupes d'étudiants volontaires et intéressés aborderont un tel sujet de façon profitable. C'est bien ce que j'ai pu constater, mais de façon très positive, deux années d'affilée avec les binômes que j'ai encadrés dans leur projet tuteuré, en 2013-2014 et 20142015. Ils auront fait tous les efforts nécessaires pour cerner et résoudre les problèmes associés à cette présentation de la TFD. En conclusion d'un travail approfondi, ils nous ont offert une présentation orale aboutie à l'image de la compréhension qu'ils ont eu du sujet.

\section{Remerciements}

L'auteur exprime ses remerciements et sa sympathie aux personnes qui suivent : Khady et Nadège, Arouna et Lahoucine. Ces étudiants ont travaillé avec enthousiasme et implication sur un projet difficile et apparaissant a priori comme austère.

\section{RÉFÉRENCES}

[1] Philippe Courmontagne, Ingénierie du signal, Théorie et pratique, Ellipses, Paris, 2005.

[2] Gérard Blanchet et Maurice Charbit, Signaux et images sous Matlab, Hermes Science, Paris, 2001

[3] Maurice Bellanger, Traitement numérique du signal, 9-ième édition, Dunod, Paris, 2012.

[4] Jean-Claude Pissondes, Voir et Entendre la Transformée de Fourier Discrète, Cetsis 2014, Besançon, 27-29 octobre 2014

[5] Jean-Claude Pissondes, Voir et Entendre la Transformée de Fourier Discrète, $3^{e}$ Workshop Pédagogique des R\&T, Saint-Pierre de la Réunion, 17-21 novembre 2014 